\title{
Erratum to: Fusibility of Agricultural Plant Waste Ash under the Conditions of High-Temperature Processing
}

\author{
M. V. Tsvetkova,*, D. N. Podlesniy ${ }^{a}$, A. Yu. Zaichenko ${ }^{a}$, \\ M. V. Salganskaya ${ }^{a}$, Yu. Yu. Tsvetkova ${ }^{a}$, \\ V. M. Freiman ${ }^{a}$, and E. A. Salgansky ${ }^{a}$ \\ a Institute of Problems of Chemical Physics, Russian Academy of Sciences, Chernogolovka, 142432 Russia \\ *e-mail: tsvetkov@icp.ac.ru
}

Received May 28, 2021; Received May 28, 2021; Received May 28, 2021

DOI: $10.1134 / \mathrm{S} 1070427221040170$

Author family name Podlesnyi should be replaced by Podlesniy. Author family name Salganskii should be replaced by Salgansky.

The original article can be found online at https://doi.org/10.1134/S1070427221030125 